\title{
Tratamiento de la hipertensión ocular y aparición de Glaucoma de Ángulo Abierto
}

The Ocular Hypertension Treatment Study: a randomized trial determines that topical ocular hypotensive medication delays or prevents the onset of primary open-angle glaucoma. Kass MA, Heuer DK, Higginbotham EJ, Johnson CA. Arch Ophthalmol. 2002;120(6):701-13.

\section{Objetivo}

Determinar la seguridad y eficacia de la medicación ocular tópica antihipertensiva, en el retardo o prevención de la aparición del Glaucoma Primario de Ángulo Abierto (GPAA).

\section{Diseño}

Ensayo clínico aleatorizado con un seguimiento de entre 6 y 6 años y medio. Los pacientes elegibles fueron aleatorizados, en forma estratificada por centro y por raza, en iguales proporciones a los grupos de tratamiento u observación, respectivamente.

\section{Lugar}

Se realizó en 22 centros universitarios privados en Estados Unidos

\section{Pacientes}

Se incluyeron pacientes entre 40 y 80 años con una presión intraocular entre 24-32 mmHg en un ojo y de entre 21-32 mmHg en el otro, con ángulos abiertos, 2 campos visuales normales y confiables por cada ojo y nervios ópticos normales. Los campos visuales y las fotografías estereoscópicas de los nervios ópticos fueron evaluados por el Centro de Lectura del estudio.

Se excluyeron pacientes con agudeza visual menor a 20/40, con cirugía ocular previa (salvo la cirugía de catarata no complicada con implante de lente intraocular), retinopatía diabética o cualquier otra patología que pudiera producir pérdida de campo visual o detrerioro del nervio óptico.

De los 3328 pacientes considerados para ser incluidos en el estudio 1636 cumplieron criterios de inclusión-exclusión y aceptaron participar: 817 fueron asignados a recibir tratamiento y 819 a observación. No hubo diferencias significativas en factores demográficos o clínicos entre los 2 grupos.

\section{Intervención}

Ni los pacientes ni los profesionales responsables de su control fueron ciegos a la intervención, aunque si lo fue la evaluación de los campos visuales y las fotografías del nervio óptico.

Los pacientes en el grupo tratado fueron medicados, utilizando 1 o más de los productos disponibles en el mercado para obtener una presión de $24 \mathrm{mmhg}$ y una reducción de la presión inicial de $20 \%$ de la misma, aunque no se requería que las presiones fuera menores a $18 \mathrm{mmhg}$.

\section{Medición de resultados principales}

El resultado principal buscado fue la aparición de glaucoma de ángulo abierto en uno o 2 ojos del mismo paciente, definido por la aparición de un defecto de campo visual reproducible en 2 o más exámenes o un deterioro clínicamente significativo del nervio óptico; en ambos casos atribuibles al glaucoma primario de ángulo abierto.

\section{Resultados principales}

\begin{tabular}{|c|c|c|c|c} 
& $\begin{array}{c}\text { Grupo Tratamiento } \\
\mathbf{n}=817(\%)\end{array}$ & $\begin{array}{c}\text { Grupo Control } \\
\mathbf{n}=819(\%)\end{array}$ & $\begin{array}{c}\text { RRR } \\
(\text { IC 95\%) }\end{array}$ & $\begin{array}{c}\text { NNT } \\
(\text { IC 95\%) }\end{array}$ \\
\hline Glaucoma & $38(4,6 \%)$ & $89(10,8 \%)$ & $57 \%(38-70)$ & $16(11-27)$ \\
\hline
\end{tabular}

Durante todo el estudio la probabilidad de desarrollar glaucoma fue significativamente inferior en el grupo medicado en comparación con el control.(Hazard Ratio 0,40;IC95\% 0,27-0,59;p < 0,0001).

\section{Conclusiones}

La medicación hipotensiva tópica fue efectiva para retardar o prevenir la aparición del glaucoma primario de ángulo abierto en individuos con hipertensión ocular.

\section{Comentario}

El estudio precedente es el ensayo clínico aleatorizado más grande realizado hasta la fecha tiene la fortaleza de su gran muestra, una cuidadosa determinación de los resultados y la valoración prospectiva y longitudinal de los casos incidentales de glaucoma. El estudio usó una definición convencional de hipertension ocular que avala su aplicabilidad pero como sucede en los ensayos con criterios de exclusión, los pacientes incluidos eran más "normales" que los típicos pacientes con hipertensión ocular de la práctica clínica. ${ }^{1}$ Igualmente se demostró que la medicación hipotensiva tópica es efectiva y segura preveniendo la evolución a GPAA, que aparece en menos de la mitad que en los controles.

Esto no implica que deban tratarse todos los pacientes con hipertensión ocular. La decisión de recomendar o no tratamiento a pacientes con hipertensión ocular debe ser acompañada de la evaluación de otros factores tales como 1) Tener presente la baja in- cidencia del GPAA en poblaciones con hipertensión ocular 2) los efectos secundarios de un tratamiento de por vida, en lo que respecta a efectos adversos y costos 3 ) El riesgo individual de desarrollar GPAA (mayor edad, alta presión intraocular y parámetros del fondo de ojo) $)^{2}$ 4) La posibilidad individual de ser ayudado por el tratamiento 5) El estado general del paciente y su expectativa de vida.

Conclusión del comentador: la medicación retarda o previene la aparición del glaucoma primario de ángulo abierto en individuos con hipertensión ocular pero no implica que deban tratarse todos los pacientes hipertensión ocular a menos que tengan moderado 0 alto riesgo de desarrollar glaucoma.

\section{Dr. Eduardo Mayorga Argañaraz [ Subjefe Servicio de Oftalmología del Hospital Italiano ]}

